\title{
Say what we mean, mean what we say
}

European Journal of Human Genetics (2006) 14, 149. doi:10.1038/sj.ejhg.5201533; published online 14 December 2005

I read with interest the publication by Cooper et al, ${ }^{1}$ 'Molecular and phenotypic expression of BeckwithWiedemann syndrome'. The article is well written, informative and documents important phenotypic information for a select group of patients. However, the study title does not accurately reflect the study methodology because the authors equate a syndrome diagnosis with a molecular defect. As stated on p.3 in the Methods section, the investigators '.. analyzed only those cases with a proven molecular (my emphasis) diagnosis of BWS.' The phrase 'molecular diagnosis of BWS' is a nonsequitur. Syndromes are clinical diagnoses, at least within the group of disorders defined by dysmorphology, and as such are defined by their specific pattern of malformation, not by their pattern on a Southern blot. To further cloud the issue, the original Cooper et al population from which the study group was culled were patients referred to the laboratory for 'suspicion' of BWS rather than from a population meeting specific diagnostic criteria. The author's concede this point in their results section, even noting two cases of isolated hemihypertrophy included in their N. They justify this approach because ' $97 \%$ of patients had at least two 'major' features of BWS...'. Unfortunately we are not given information as to which two major defects were present. Should a patient, for instance, with an umbilical hernia and a renal defect be considered to have BWS? As a result of not using specific diagnostic criteria and of reporting results only from patients with a molecular alteration, the authors have not categorized the molecular and phenotypic expression of BWS syndrome as the title indicates, but instead have categorized the phenotypic expression of a series of patient's with specific genetic and epigenetic alterations of the $11 \mathrm{p} 15.5$ region, and have done so quite nicely at that. The distinction is an important one. The era of gene discovery is winding down as we move into an exciting period of the search for modifying elements of human inheritance. Answering the question as to why two people with an identical genetic or epigenetic alteration have two distinctively different phenotypes is a next step in connecting the dots between gene mutation and pathogenesis. For example, we have recently showed that $1 / 3$ of isolated hemihyperplasia (IH) patients have the same Lit1 and/or H19 methylation abnormalities as BWS. ${ }^{2}$ However, we have not gone back to those patients and labeled them with BWS. Clearly, lumping IH and other 'suspicious' BWS cases together and classifying them as BWS after a 'BWS' genetic alteration is found as Cooper et al did in this study dilutes the opportunity to understand why they are different. Their study methodology stands on its own and offers important information, so the title should say what it means and mean what it says.

\section{RA Martin ${ }^{1}$ \\ ${ }^{1}$ Washington University School of Medicine, St Louis, Missouri 63105, USA E-mail: martin_r@kids.wustl.edu}

\footnotetext{
References

1 Cooper WN, Luharia A, Evans GA et al: Molecular subtypes and phenotypic expression of Beckwith-Wiedemann syndrome. Eur J Hum Genet 2005; 13: 1025-1032.

2 Martin RA, Grange DK, Zehnbauer B, DeBaun MR: LIT1 and H19 methylation defects in isolated hemihyperplasia. Am J Med Genet $A$ 2005; 134: 129-131.
}

\section{Reply to Dr Martin}

European Journal of Human Genetics (2006) 14, 149-150. doi:10.1038/sj.ejhg.5201555

Dr Martin raises important points regarding the nosology of syndrome diagnosis. Clearly there are instances when the identification of a molecular marker allows the diagnosis of a genetic disorder in individuals who do not satisfy the clinical diagnostic criteria. For example, the identification of a VHL or RET gene mutation in an individual with phaeochromocytoma has important implications for prognosis and most clinicians would then 
label that individual as having von Hippel-Lindau disease or Multiple Endocrine Neoplasia Type 2 even if no other clinical features of these disorders were present. Such examples are not uncommon and blur the distinctions between clinical and laboratory criteria for syndrome diagnosis. In addition, it is not uncommon for the diagnostic criteria for a specific syndrome to be redefined after a molecular test becomes available. Beckwith-Wiedemann syndrome (BWS) often poses diagnostic problems because there are no generally agreed clinical diagnostic criteria. Thus, originally we suggested strict diagnostic criteria (either three 'major features (macroglossia, abdominal wall defect and pre- or postnatal overgrowth) or two of these plus three or more minor features (ear lobe creases or posterior helical ear pits, facial naevus flammeus, hypoglycaemia, nephromegaly and hemihypertrophy), whereas others have used broader definitions (two or more of ear pits or creases, macroglossia, macrosomia, hypoglycaemia in the first month of life and anterior abdominal wall defect and a diagnosis of BWS by a physician). ${ }^{1,2}$ Hence, the incorporation of molecular genetic testing results into BWS diagnostic criteria would be a significant advance towards formulating commonly agreed diagnostic criteria. In our study, we noted that $97 \%$ of our patients with a molecular abnormality had two or more 'major features' (macroglossia, abdominal wall defect and pre- or postnatal overgrowth). ${ }^{3}$ Of the remaining children, all except one patient had one major and at least one 'minor feature' (ear pits or creases, neonatal hypoglycaemia or facial naevus flammeus). One patient with loss of methylation at
KvDMR1 (imprinting centre 2 defect) was reported as having isolated hemihypertrophy only. Whether this patient should be labelled as having BWS might be debated, but their inclusion or exclusion in our study would not materially affect the conclusions of our research. Thus, although a negative molecular investigation does not exclude the diagnosis of BWS, in many cases, molecular genetic testing is a useful diagnostic and prognostic indicator and molecular tests should be incorporated into BWS diagnostic criteria.

Eamonn R Maher

Section of Medical and Molecular Genetics, University of Birmingham, Birmingham, UK

Correspondence: Professor ER Maher, Section of Medical and Molecular Genetics, University of Birmingham, Edgbaston, Birmingham, UK. Tel: + 44121627 2741;

Fax: + 44121627 2618;

E-mail: e.r.maher@bham.ac.uk

\section{References}

1 Elliott M, Maher ER: Beckwith-Wiedemann syndrome. J Med Genet 1994; 31: 560-564.

2 Wangler MF, An P, Feinberg AP, Province M, Debaun MR: Inheritance pattern of Beckwith-Wiedemann syndrome is heterogeneous in 291 families with an affected proband. Am J Med Genet A 2005; 137: 16-21.

3 Cooper WN, Luharia A, Evans GA et al: Molecular subtypes and phenotypic expression of Beckwith-Wiedemann syndrome. Eur J Hum Genet 200; 13: 1025-1032. 